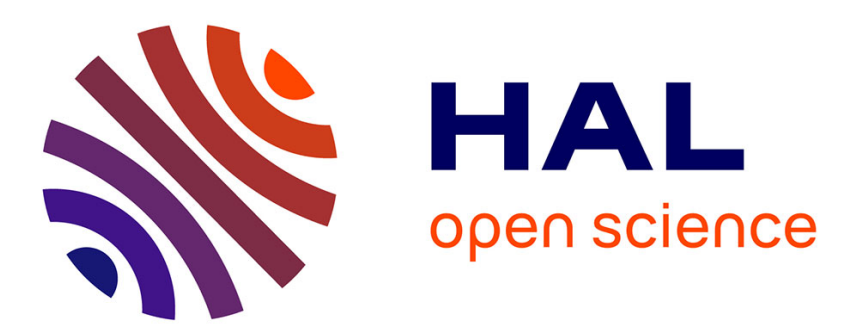

\title{
Application of the transmission line matrix method for outdoor sound propagation modelling - Part 1: Model presentation and evaluation
}

Gwenaël Guillaume, Pierre Aumond, Benoit Gauvreau, Guillaume Dutilleux

\section{To cite this version:}

Gwenaël Guillaume, Pierre Aumond, Benoit Gauvreau, Guillaume Dutilleux. Application of the transmission line matrix method for outdoor sound propagation modelling - Part 1: Model presentation and evaluation. Applied Acoustics, 2014, 76, pp.113-118. 10.1016/j.apacoust.2013.07.011 . hal-00949035

\section{HAL Id: hal-00949035 \\ https://hal.science/hal-00949035}

Submitted on 19 Feb 2014

HAL is a multi-disciplinary open access archive for the deposit and dissemination of scientific research documents, whether they are published or not. The documents may come from teaching and research institutions in France or abroad, or from public or private research centers.
L'archive ouverte pluridisciplinaire HAL, est destinée au dépôt et à la diffusion de documents scientifiques de niveau recherche, publiés ou non, émanant des établissements d'enseignement et de recherche français ou étrangers, des laboratoires publics ou privés. 


\title{
Application of the Transmission Line Matrix method for outdoor sound propagation modelling - Part 1: Model presentation and evaluation
}

\author{
Gwenaël Guillaume ${ }^{\mathrm{a}}$, Pierre Aumond ${ }^{\mathrm{a}}$, Benoit Gauvreau ${ }^{\mathrm{a}}$, Guillaume \\ Dutilleux ${ }^{b}$ \\ ${ }^{a}$ Institut Français des Sciences et Technologies des Transports, de l'Aménagement et des \\ Réseaux, Département Infrastructures et Mobilité, Route de Bouaye, B.P. CS4, 44344 \\ Bouguenais Cedex, France \\ ${ }^{b}$ Laboratoire Régional des Ponts et Chaussées de Strasbourg, ERA 32, 11 rue Jean \\ Mentelin, BP 9, 67035 Strasbourg Cedex 2, France
}

\section{Abstract}

The present paper deals with an original time-domain approach applied to outdoor sound propagation under meteorological effects. The Transmission Line Matrix method, based on the Huygens' principle, had already been validated over impedant grounds and complex topography. The presented formulation proposes to take into account meteorological effects (wind speed and temperature) through the relative sound speed. The necessary wavefront direction is determined through the calculation of the averaged intensity vector direction. A good agreement is found between simulations of both the transmission line matrix and parabolic equation methods. A relevant use of the method is shown in the framework of environmental acoustics and initial applications are proposed in Part 2.

Keywords:

Email address: guillaume.gwenael@neuf.fr (Gwenaël Guillaume) 
Time-domain methods, Transmission Line Matrix model, Meteorological effects, Outdoor sound propagation

\section{Introduction}

Many numerical models have been developed in order to simulate meteorological effects on outdoor sound propagation. One can cite analytical formulations such as geometrical methods (e.g. ray or gaussian beam tracing approaches) [1,2] or numerical models based on the resolution of the wave equation. Among these latter models, the Parabolic-Equation (PE) based method has been widely used for this purpose $[2,3,4,5]$. Over the last decade, with the increasing power of computational resources, time-domain methods have also been developed and applied successfully in environmental acoustics $[6,7,8,9]$. The most popular time-domain approach is undoubtedly the finite-difference in the time-domain (FDTD) method. Dragna has investigated for instance sound propagation over a $100 \mathrm{~m}$ distance within a realistic context in a frequency range between $100 \mathrm{~Hz}$ and $2 \mathrm{kHz}$ [10]. An alternative time-domain method is the Transmission Line Matrix (TLM) approach [11]. This model seems very promising for describing complex outdoor sound propagation yet has not been used extensively. In order to incorporate the atmospheric effects, Hofmann has proposed an interesting formulation, which is however limited to temperature effects [12]. A first attempt to define a TLM scheme with unidirectional mean flow (e.g. wind field) into the TLM grid has been provided by Kagawa and his co-workers, but its method is only successful for single wind speed direction effects [13]. In this paper, the approach, inspired by a method proposed by Dutilleux [14], consists of mod- 
ifying the sound speed at each point of the discretized domain as a function of the temperature and wind speed projection on the wave front direction.

This paper aims to present the integration of meteorological effects in the TLM model and then its comparison with a PE model in academic cases. The associated paper (part 2) addresses its validation by comparison with results stemming from Lannemezan's 2005 experimental campaign [15]. The wind and temperature fields are obtained from the meso-scale meteorological model Meso-NH $[16,17]$.

The first section presents the TLM model. In section 2 the formulation taking into account wind speed and temperature effects is described. The last section proposes evaluating the ability of the TLM to treat outdoor sound propagation problems through a comparison with PE results in two academic cases.

\section{TLM modelling}

The TLM method is based on the Huygens' principle, which states that a wavefront consists of a set of secondary sources radiating spherical wavelets whose envelopes can be broken down into a new generation of secondary sources as well. Hofmann has shown the equivalence of this approach with the resolution of the discretized wave equation [12]. Other authors have also derived the two-dimensional homogeneous cartesian formulation of TLM from a Lattice Boltzmann model by removing nonlinear terms, in choosing a suitable viscosity and selecting a square grid [18].

The TLM statement allows describing sound propagation through both a spatial and temporal discretization of the medium as well as the propa- 
gation phenomena. This concept is numerically conveyed by replacing the propagation medium with a transmission lines network, through which sound propagates in the form of sound pulses. Thereby, as shown in Fig. 1, each junction, or node, links $N=4$ or $N=6$ transmission lines to each other in two dimensions (2D) or three dimensions (3D) respectively. Thereafter, the number of dimensions is called $d$ such that $d=2$ and $d=3$ for creating a 2D and 3D model respectively. An additive branch, of index $N+1$, is inserted at each node of the transmission line network, in order to consider the inhomogeneities of the propagation medium (i.e. branch 7 in Fig. 1). According to the TLM concept, sound propagates in the form of pulses. Thus, incident and scattered pulses are considered at each transmission lines junction and time increment. The propagation medium is discretized by means of a uniform cartesian meshing of mesh size $(\Delta l)^{d}$, with $\Delta l$ being the spatial step such that:

$$
\Delta l \leqslant \frac{\lambda \sqrt{d}}{10}
$$

with $\lambda$ the minimal wavelength of the simulation.

The scattered pulses at time increment $t$ and node of discrete coordinates r such that

$$
\mathbf{r}= \begin{cases}(i, j) & \text { for } d=2(i . e . \text { in } 2 \mathrm{D}), \\ (i, j, k) & \text { for } d=3(i . e . \text { in 3D), }\end{cases}
$$

are related with the incident pulses at this node at the same time iteration by the following matrix relation:

$$
{ }_{t} \mathbf{S}_{\mathbf{r}}={ }_{t} \mathbf{D}_{\mathbf{r}} \times{ }_{t} \mathbf{I}_{\mathbf{r}},
$$

where ${ }_{t} \mathbf{I}_{\mathbf{r}}$ and ${ }_{t} \mathbf{S}_{\mathbf{r}}$ are the vectors composed of the incident pulses ${ }_{t} I_{\mathbf{r}}^{n}$ and scattered pulses ${ }_{t} S_{\mathbf{r}}^{n}$ through each transmission line $n(n=1$ to $N+1)$ 

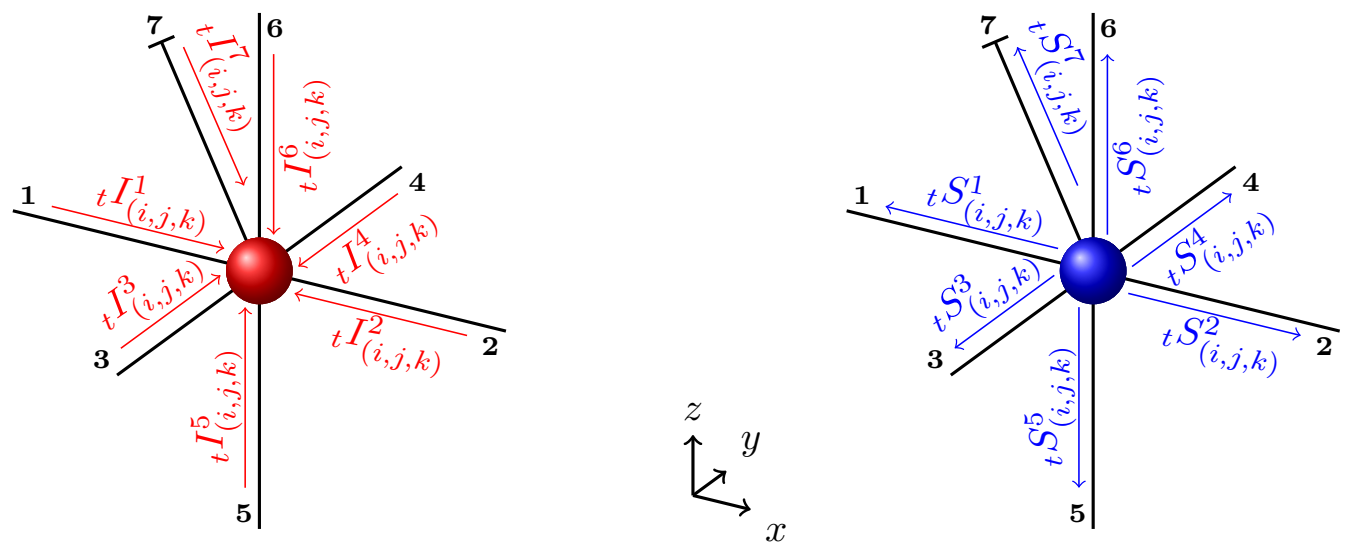

Figure 1: Representation of the incident (left) and the scattered (right) pulses at node $(i, j, k)$ in $3 \mathrm{D}$.

respectively. ${ }_{t} \mathbf{D}_{\mathbf{r}}$ is a $(N+1) \times(N+1)$ scattering matrix given by:

$$
{ }_{t} \mathbf{D}_{r}=\frac{2}{{ }_{t} \eta_{\mathbf{r}}+2 d}\left[\begin{array}{ccccc}
{ }_{t} a_{\mathbf{r}} & 1 & \cdot & 1 & { }_{t} \eta_{\mathbf{r}} \\
1 & { }_{t} a_{\mathbf{r}} & \cdot & 1 & { }_{t} \eta_{\mathbf{r}} \\
\cdot & \cdot & \cdot & \cdot & \cdot \\
1 & 1 & \cdot & { }_{t} a_{\mathbf{r}} & \eta_{\mathbf{r}} \\
1 & 1 & \cdot & 1 & { }_{t} b_{\mathbf{r}}
\end{array}\right],
$$

with

$$
{ }_{t} a_{\mathbf{r}}=-\frac{{ }_{t} \eta_{\mathbf{r}}+2(d-1)}{2}
$$

and

$$
{ }_{t} b_{\mathbf{r}}=\frac{{ }_{t} \eta_{\mathbf{r}}-2 d}{2}
$$

where ${ }_{t} \eta_{\mathbf{r}}$ allows locally modifying, and if needed during the simulation, the sound speed in the propagation medium. In other words, this term is used in order to model an inhomogeneous atmosphere and is defined in section 3.1. 
Finally, the nodal pressure is written as a combination of all incident pulses, i.e.:

$$
{ }_{t} p_{\mathbf{r}}=\frac{2}{{ }_{t} \eta_{\mathbf{r}}+2 d}\left(\sum_{n=1}^{N-1}{ }_{t} I_{\mathbf{r}}^{n}+{ }_{t} \eta_{\mathbf{r}} I_{\mathbf{r}}^{N}\right) .
$$

In addition, the scattered pulses from nodes adjacent to node $(i, j, k)$ at time increment $t$ become the incident pulses to this node at the next time iteration $t+\Delta t$, with $\Delta t$ representing the time step defined by:

$$
\Delta t=\frac{\Delta l}{\sqrt{d} c_{0}},
$$

with $c_{0}$ the adiabatic sound speed. This diffusion process is governed by connection laws depicted in Fig. 2 such as:

$$
{ }_{t+\Delta t} I_{\mathbf{r}}^{n}={ }_{t} S_{\mathbf{r}_{n}^{ \pm}}^{m}
$$

and

$$
{ }_{t+\Delta t} I_{\mathbf{r}}^{N}={ }_{t} S_{\mathbf{r}}^{N},
$$

with

$$
\left\{\begin{array}{c}
n \\
\mathbf{r}_{n}^{ \pm}
\end{array}\right\}=\left\{\begin{array}{c}
\left\{\begin{array}{c}
m-1 \\
\mathbf{r}^{-}
\end{array}\right\} \text {if } m \text { is even } \\
\left\{\begin{array}{c}
m+1 \\
\mathbf{r}^{+}
\end{array}\right\} \text {if } m \text { is odd }
\end{array}\right.
$$

and

$$
\mathbf{r}_{n}^{ \pm}=\left\{\begin{array}{l}
(i \pm 1, j, k), \text { for } n=1 \text { or } 2 \\
(i, j \pm 1, k), \text { for } n=3 \text { or } 4 \\
(i, j, k \pm 1), \text { for } n=5 \text { or } 6
\end{array}\right.
$$




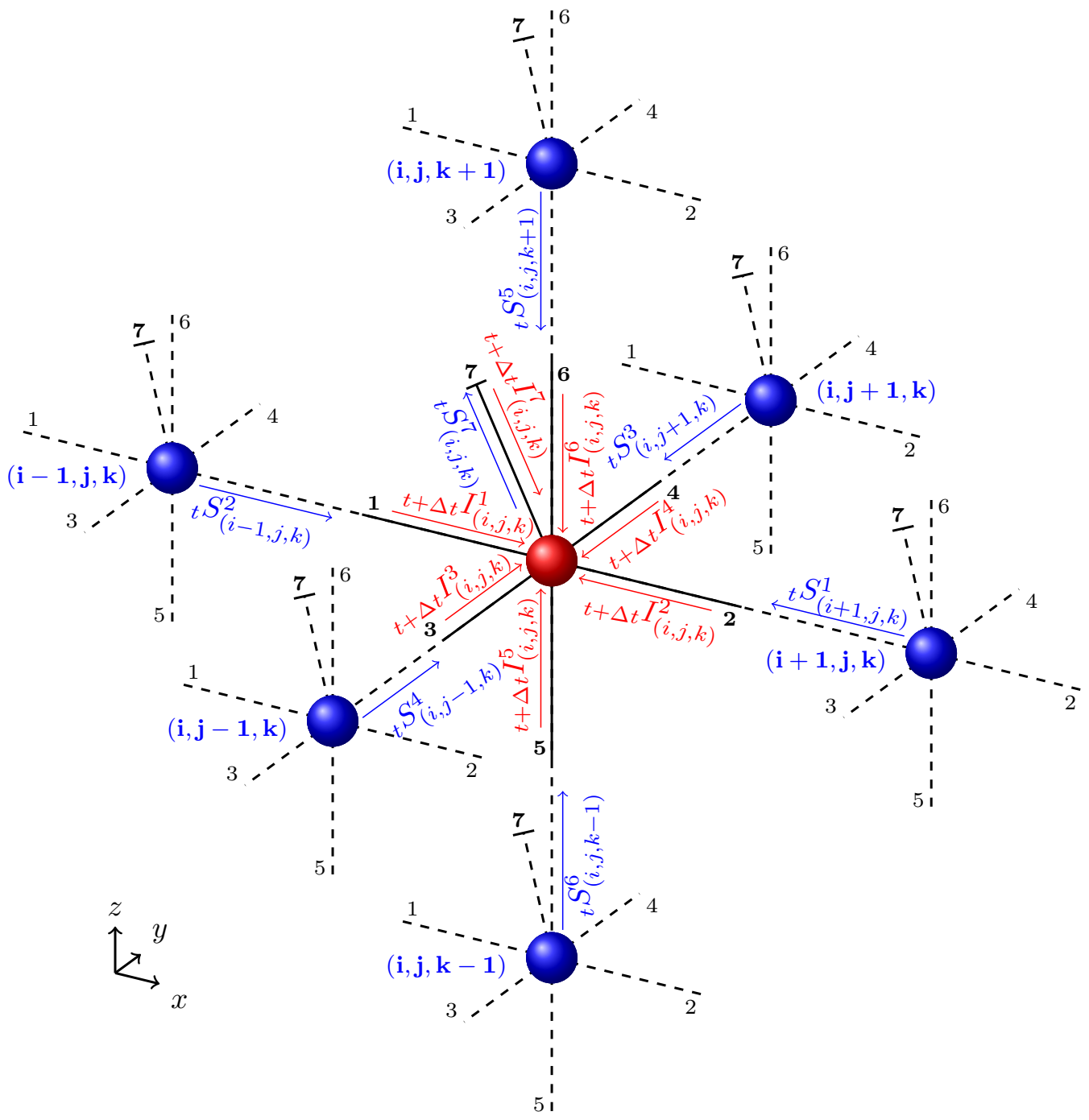

Figure 2: Representation of the connection laws through the main transmission lines $(n=1$ to $N)$ at the node $(i, j, k)$ in $3 \mathrm{D}$.

Boundaries are implemented in the TLM model at a distance $\Delta l / 2$ from the nearest node in order to ensure the synchronism of sound pulses. They can be characterized by a reflection coefficient in pressure [11] or by an 
impedance boundary condition [19]. In addition, absorbing layers can be introduced in order to model an unbounded propagation medium as depicted in Ref. [20].

\section{TLM formulation for acoustic propagation in a meteorological field}

Regarding outdoor sound propagation, the temperature and wind fields combine to produce local variations of sound speed. The literature review above has shown that the implementation of thermal gradients is straightforward in TLM since sound speed is defined at the local level, whereas a suitable implementation for wind gradients is still underdeveloped. In order to address the general case of outdoor sound propagation, both thermal and wind effects must be taken into account simultaneously.

In order to allow for wind speed gradients in the TLM model, the approach developed in this section satisfies the previous requirement since it is based on the so-called effective sound speed. This approach requires knowl-

edge of the local direction of the wavefront. A proof of concept of this approach for the TLM method has been proposed by one of the authors in Ref. [14].

\subsection{Effective sound speed}

The effective sound speed is expressed as follows [2]:

$$
{ }_{t} c_{\mathrm{eff}_{\mathbf{r}}}=\sqrt{\gamma R_{t} T_{\mathbf{r}}}+{ }_{t} \vec{u}_{\mathbf{r}} \cdot{ }_{t} \vec{n}_{\mathbf{r}}
$$

where $\gamma=1.41$ and $R=287 \mathrm{~J} . \mathrm{kg}^{-1} \cdot \mathrm{K}^{-1}$, representing the specific heat ratio and the perfect gas constant for air respectively. ${ }_{t} T_{\mathbf{r}}$ is the temperature, ${ }_{t} \vec{u}_{\mathbf{r}}$ 
is the wind speed vector and ${ }_{t} \vec{n}_{\mathbf{r}}$ is the wavefront direction. The sound speed at a point $\mathbf{r}$ of the TLM grid verifies [21]:

$$
{ }_{t} c_{\mathrm{TLM}_{\mathbf{r}}}=c_{0} \sqrt{\frac{2}{{ }_{t} \eta_{\mathbf{r}}+2 d}}
$$

So, if one sets ${ }_{t} c_{\mathrm{eff}_{\mathrm{r}}}={ }_{t} c_{\mathrm{TLM}_{\mathbf{r}}}$, solving for the inhomogeneity parameter $\eta$ leads to:

$$
{ }_{t} \eta_{\mathbf{r}}=\frac{2}{\sqrt{\gamma R_{t} T_{\mathbf{r}}}+{ }_{t} \vec{u}_{\mathbf{r}} \cdot{ }_{t} \vec{n}_{\mathbf{r}}} \frac{\Delta l^{2}}{\Delta t^{2}}-2 d .
$$

In order to ensure that $\eta \geqslant 0$, the adiabatic sound speed $c_{0}$ must verify:

$$
c_{0} \geqslant \sqrt{\gamma R T_{\max }+\|\vec{u}\|_{\max }}
$$

where the subscript max is computed over the whole simulated domain and simulation duration.

\subsection{Wavefront direction}

Vector $\vec{n}$ defines the direction of the wavefront. This information is not readily available in the TLM approach, yet it can be approximated by a quantity relating the instantaneous sound intensity vector:

$$
{ }_{t} \vec{I}_{\mathbf{r}}={ }_{t} p_{\mathbf{r}} \cdot{ }_{t} \vec{v}_{\mathbf{r}}
$$

where ${ }_{t} \vec{v}_{\mathbf{r}}$ is the local instantaneous velocity defined in [22]:

$$
{ }_{t} \vec{v}_{\mathbf{r}}=\frac{1}{\rho_{0} c_{0}}\left({ }_{t} S_{\mathbf{r}}^{m}-{ }_{t} S_{\mathbf{r}}^{n}\right)
$$

where $m$ is even (see Eq. 11).

A purely local definition of the sound intensity is not suitable as an approximation for $\vec{n}$ because it is influenced by the reactive intensity which 
does not propagate. In order to eliminate the reactive intensity, a possible approach is to average intensity over space, for instance over a onewavelength-size domain. As a first approximation and since meteorological effects (e.g. wind-induced refraction) are a long-range phenomenon, this averaging approach is only valid far away from the source. Thus, the average intensity vector corresponds with the active intensity vector and is computed as follows:

$$
{ }_{t} \vec{I}_{a v g, \mathbf{r}}=\frac{1}{(2 a+1)^{d}} \sum_{r_{n}-a}^{r_{n}+a}{ }_{t} \vec{I}_{r_{n}},
$$

where $a$ sets the span of the average around $r_{n}$.

From Eq. (19), one defines:

$$
{ }_{t} \vec{n}_{\mathbf{r}}=\frac{\vec{I}_{a v g, \mathbf{r}}}{\left\|\vec{I}_{a v g, \mathbf{r}}\right\|} .
$$

The angle $\theta$ between the wavefront and the wind directions can be defined in 2D as:

$$
{ }_{t} \theta_{\mathbf{r}}=\frac{{ }_{t} \vec{u}_{\mathbf{r}} \cdot{ }_{t} \vec{n}_{\mathbf{r}}}{\left\|\vec{u}_{\mathbf{r}}\right\| \times\left\|{ }_{t} \vec{n}_{\mathbf{r}}\right\|}
$$

Fig. 3 illustrates the wave direction and the angle $\theta$ obtained without averaging (i.e. the vector ${ }_{t} \vec{n}_{\mathbf{r}}$ corresponds with the normalized instantaneous intensity vector) and by averaging the variables with a spatial span $a=10$. The reactive intensity contribution appears clearly when no averaging is applied, i.e. where the sign of the sound pressure changes. Spatial averaging significantly improves the determination of the wavefront propagation direction. 

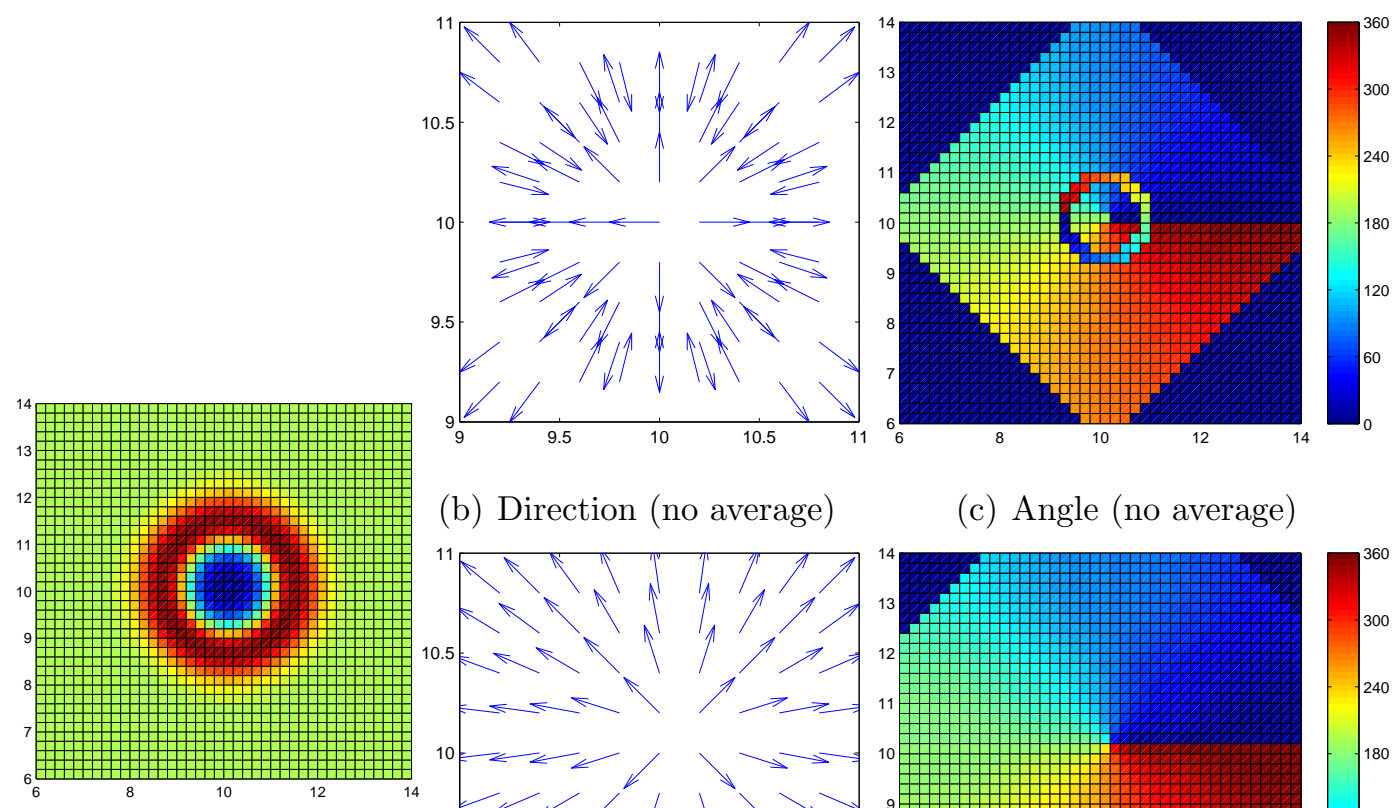

(b) Direction (no average)

(c) Angle (no average)

(a) Wavefront

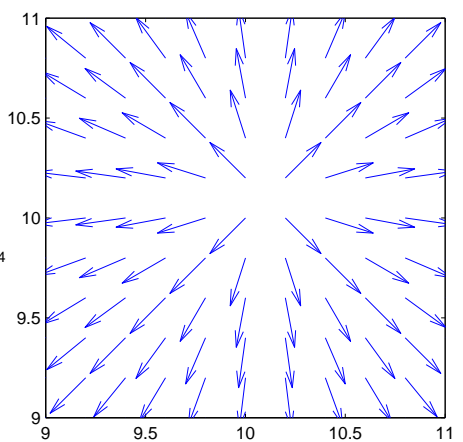

(d) Direction (spatial average)

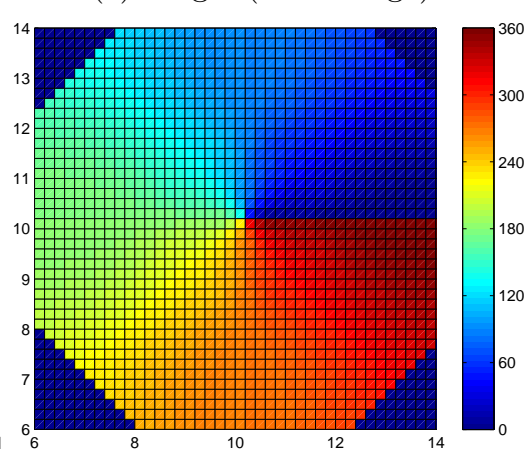

(e) Angle (spatial average)

Figure 3: Computation of the wave propagation direction and of the angle $\theta$. Representation of (a) the wavefront, and of the wave propagation direction and of the angle $\theta$ obtained from (b)-(c) the instantaneous acoustic intensity and (d)-(e) the averaged acoustic intensity with a spatial span $a=10$. 


\section{Comparison with $\mathrm{PE}$ results}

In order to evaluate the ability of the proposed formulation to take into account meteorological effects (through wind and temperature fields) in the TLM model, 2D simulation results are compared with results obtained with the Parabolic Equation (PE) model [3], which is widely used for this purpose.

Three simulation results are presented. The source is located alternatively at $0.15 \mathrm{~m}, 2.05 \mathrm{~m}$ and $5.05 \mathrm{~m}$ from the ground. First, the sound attenuation levels are compared over a line of receivers located at a height of $2.05 \mathrm{~m}$ and at a fixed frequency of $200 \mathrm{~Hz}$. Then, the attenuation spectra are given at $150 \mathrm{~m}$ from the source. The upper frequency bound is fixed in order to limit the computation cost.

The simulation presented in Fig. 4 is performed over a rigid ground through an atmosphere characterized by a linear vertical gradient of temperature $\left(d T / d z=0.35^{\circ} \mathrm{Cm}^{-1}\right)$ and a wind field set to zero. The sound attenuations levels are very similar. The discrepancy in the first $50 \mathrm{~m}$ can be explained by the PE limit at short distances from the source [2]. The depth peak differences are due to the sensitivity and numerical uncertainties of the two models. This academic case allows us to validate the effective sound speed approach without any air flow $\left(c_{\mathrm{eff}_{\mathbf{r}}}=\sqrt{\gamma R T_{\mathbf{r}}}\right)$.

Since the meteorological effets tend to increase ground effects in the case of downward refraction conditions, the second simulation proposes to consider an impedant ground defined by the Miki impedance model [23] with an air flow resistivity $\sigma=100 \mathrm{kN} . \mathrm{s} . \mathrm{m}^{-4}$. In addition, the effective sound speed field is calculated for a homogeneous temperature field $\left(\mathrm{T}=20^{\circ} \mathrm{C}\right)$ and for a realistic vertical wind gradient of $0.2 \mathrm{~s}^{-1}$. Thus, it allows evaluating the 


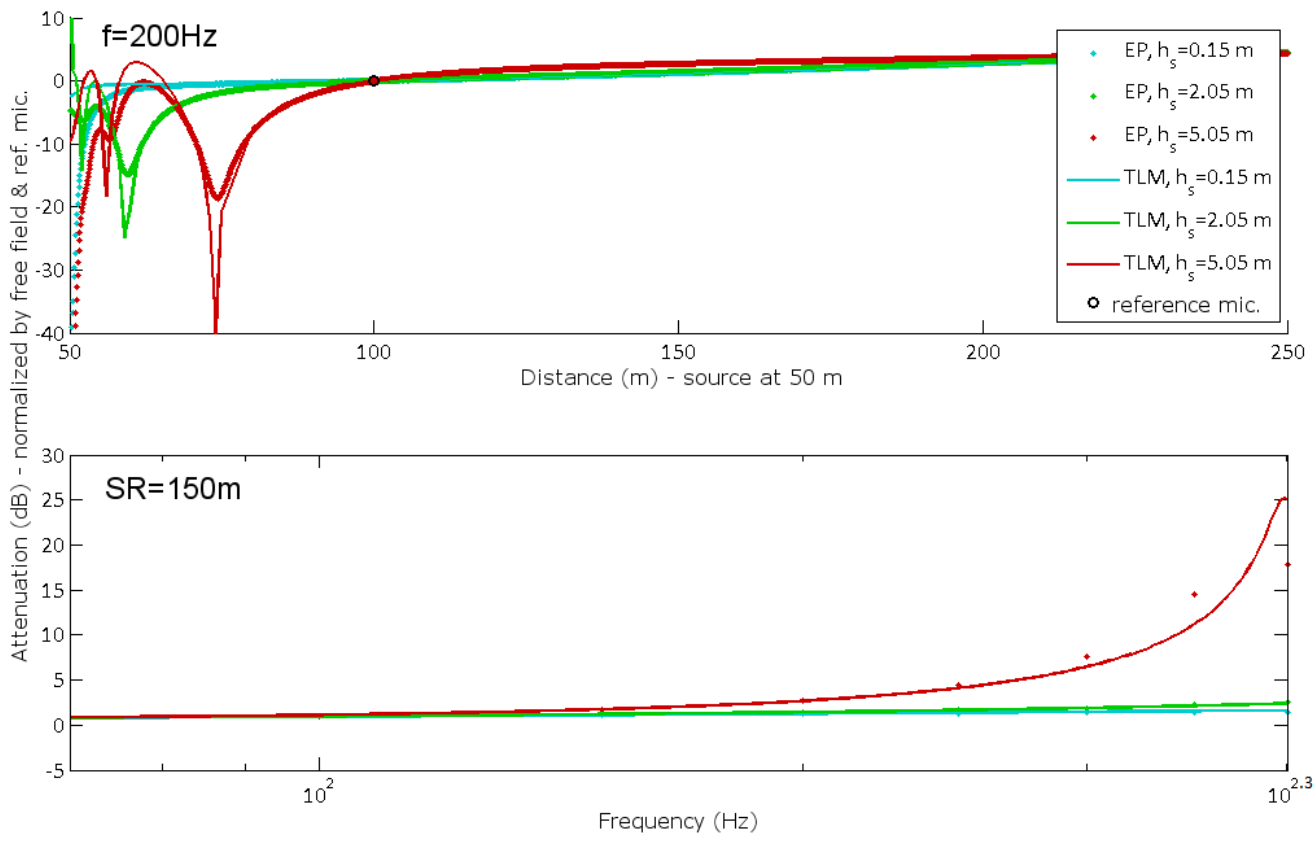

Figure 4: Comparison of the TLM model simulations (lines) with the PE ones (dots) for the sound propagation over a rigid ground with a linear temperature gradient such as $d T / d z=0.35^{\circ} \mathrm{Cm}^{-1}$. The source is located at $0.15 \mathrm{~m}$ (blue), $2.05 \mathrm{~m}$ (green) and $5.05 \mathrm{~m}$ (red) from the ground.

formulation proposed in Section 3.2. Fig. 5 shows the good agreement observed between the two model results. The frequency $(\mathrm{PE})$ and time-domain (TLM) models give very similar sound level attenuations, both according to distance and frequency. In addition, the interference patterns are almost identically represented by the two models, except close to the source due to PE limitations in the near field. 


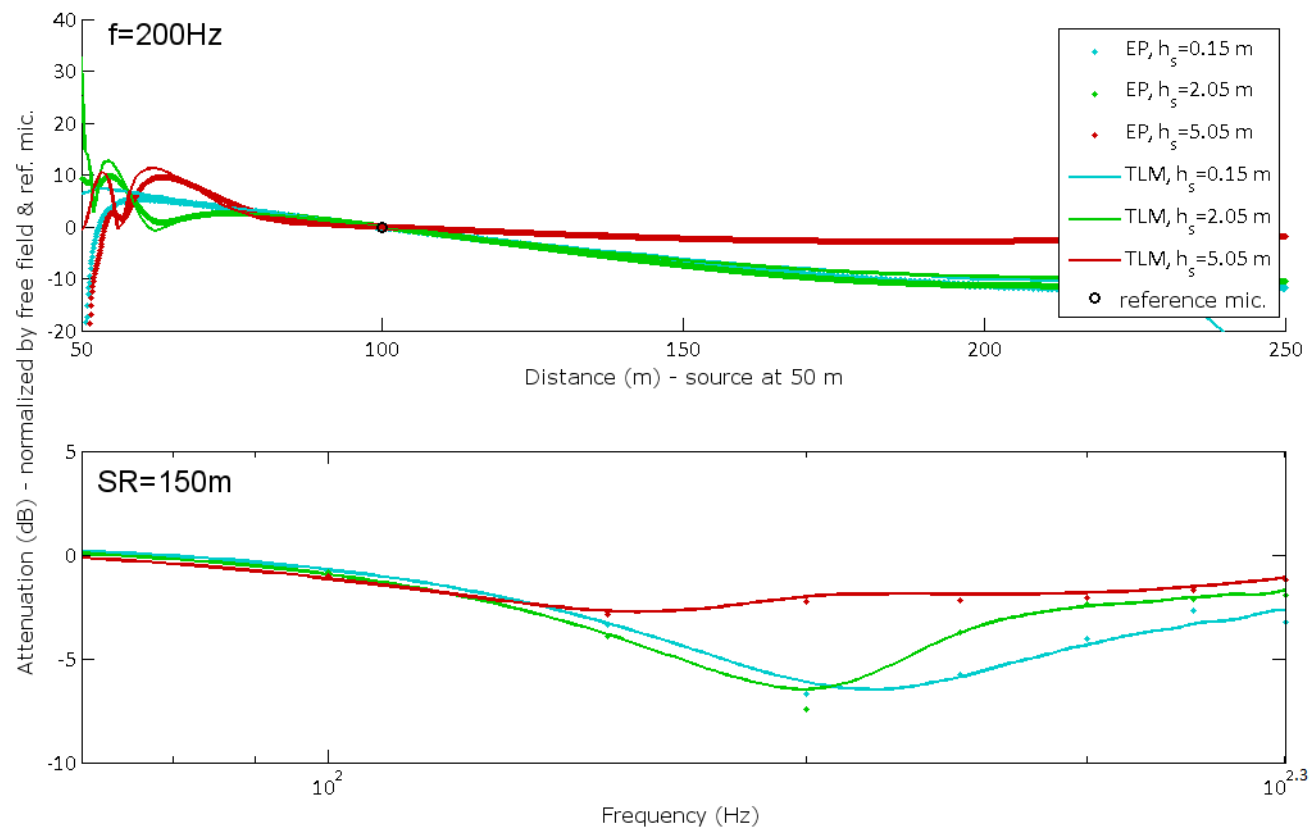

Figure 5: Comparison of the TLM model simulations (lines) with the PE ones (dots) for the sound propagation over a rigid ground with a linear wind gradient such as $d U / d z=0.2 \mathrm{~s}^{-1}$. The source is located at $0.15 \mathrm{~m}$ (blue), $2.05 \mathrm{~m}$ (green) and $5.05 \mathrm{~m}$ (red) from the ground.

\section{Conclusion}

This paper deals with the time-domain modelling of outdoor sound propagation by using a TLM model. The proposed formulation integrates meteorological variables (i.e. wind and temperature fields) through the definition of the relative sound speed. It must be noted that the use of the effective sound speed limits this model to far-field simulations. The capability of the TLM model in the context of outdoor sound propagation under meteorological effects is demonstrated through a comparison with PE simulations. 
In the second part of this paper, TLM simulations are performed in realistic cases (with measured meteorological fields as input data) using the approach presented in this part. Thus, numerical sound predictions are compared with experimental data obtained under various meteorological conditions.

\section{References}

[1] V. E. Ostashev, Acoustics in Moving Inhomogeneous Media, E \& FN SPON, ISBN 0-419-22430-0, London, England, 1997.

[2] E. M. Salomons, Computational Atmospheric Acoustics, Kluwer Academic, ISBN 9781402003905, Dordrecht, The Netherlands, 2001.

[3] B. Lihoreau, B. Gauvreau, M. Bérengier, P. Blanc-Benon, I. Calmet, Outdoor sound propagation modeling in realistic environments: Application of coupled parabolic and atmospheric models, The Journal of the Acoustical Society of America 120 (1) (2006) 110-119.

[4] T. Van Renterghem, D. Botteldooren, P. Lercher, Comparison of measurements and predictions of sound propagation in a valley-slope configuration in an inhomogeneous atmosphere, Journal of the Acoustical Society of America 121 (2007) 2522-2533.

[5] B. Cotté, P. Blanc-Benon, Estimates of the relevant turbulent scales for acoustic propagation in an upward refracting atmosphere, Acta Acustica united with Acustica 93 (6) (2007) 944-958. 
[6] B. Chopard, P. Luthi, Lattice boltzmann computations and applications to physics, Theoretical Computer Science 217 (1) (1999) 115-130.

[7] R. Blumrich, D. Heimann, A linearized eulerian sound propagation model for studies of complex meteorological effects, Journal of the Acoustical Society of America 112 (2) (2002) 446-455.

[8] V. Ostashev, D. Wilson, L. Liu, D. Aldridge, N. Symons, D. Marlin, Equations for finite-difference, time-domain simulation of sound propagation in moving inhomogeneous media and numerical implementation, Journal of the Acoustical Society of America 117 (2) (2005) 503-517. doi:10.1121/1.1841531.

[9] M. Hornikx, R. Waxler, J. Forssén, The extended fourier pseudospectral time-domain method for atmospheric sound propagation., The Journal of the Acoustical Society of America 128 (4) (2010) 1632-46.

[10] D. Dragna, Modélisation par une approche temporelle de la propagation acoustique en milieu extérieur : traitement de frontières complexes et validation sur site ferroviaire, Ph.D. thesis, Ecole Centrale de Lyon, Lyon, France (2011).

[11] Y. Kagawa, T. Tsuchiya, B. Fujii, K. Fujioka, Discrete Huygens' model approach to sound wave propagation, Journal of Sound and Vibration 218 (3) (1998) 419-444.

[12] J. Hofmann, K. Heutschi, Simulation of outdoor sound propagation with a transmission line matrix method, Applied Acoustics 68 (2) (2007) 158172. 
[13] Y. Kagawa, T. Tsuchiya, T. Hara, T. Tsuji, Discrete Huygens' modelling simulation of sound wave propagation in velocity varying environments, Journal of Sound and Vibration 246 (3) (2001) 419-439.

[14] G. Dutilleux, Applicability of TLM to wind turbine noise prediction, in: Second International Meeting on Wind Turbine Noise, Lyon, France, 2007.

[15] F. Junker, B. Gauvreau, D. Ecotière, C. Cremezi-Charlet, P. BlancBenon, Meteorological classification for environmental acoustics - Practical implications due to experimental accuracy and uncertainty, in: International Congress on Acoustics (ICA), Madrid, Spain, 2007.

[16] J. Lafore, J. Stein, N. Asencio, P. Bougeault, V. Ducrocq, J. Duron, C. Fischer, P. Hereil, P. Mascart, J. Pinty, J. Redelsperger, E. Richard, J. V. d. Arellano, The Meso-nH atmospheric simulation system. Part I: Adiabatic formulation and control simulations, Annales Geophysicae 16 (1998) 90-109.

[17] P. Aumond, C. Lac, V. Masson, B. Gauvreau, M. Bérengier, Including drag effects of canopies: Real case large-eddy simulation studies, Boundary Layer Meteorology 146 (1) (2013) 65-80.

[18] M. Martin, B. Chopard, Low Cost Parallelizing: A Way to be Efficient, in: Vector And Parallel Processing - VECPAR98, Lecture Notes in Computer Science, Springer, Berlin, 1999, pp. 522-533.

[19] G. Guillaume, J. Picaut, G. Dutilleux, B. Gauvreau, Time-domain impedance formulation for transmission line matrix modelling of out- 
door sound propagation, Journal of Sound and Vibration 330 (26) (2011) 6467-6481. doi:10.1016/j.jsv.2011.08.004.

[20] G. Guillaume, J. Picaut, A simple absorbing layer implementation for transmission line matrix modeling, Journal of Sound and Vibration 332 (19) (2013) 4560-4571. doi:10.1016/j.jsv.2013.04.003.

[21] G. Guillaume, Application of the TLM method for the sound propagation modeling in urban area (Application de la méthode TLM à la modélisation de la propagation acoustique en milieu urbain), Ph.D. thesis, Doctoral School SPIGA Science Engineering, Geosciences, Architecture (October 2009).

[22] Y. Kagawa, T. Tsuchiya, B. Fujii, M. Takeuchi, Discrete Huygens' model approach to sound wave propagation - Reverberation in a room. Sound source identification and tomography in time reversal, Journal of Sound and Vibration 225 (1) (1999) 61-78.

[23] Y. Miki, Acoustical properties of porous materials - Modifications of Delany-Bazley models, The Journal of the Acoustical Society of Japan (E) 11 (1) (1990) 19-24. 